\title{
Physical Parameter Identification Algorithm Modeling for Complex Microsystem via Triangulation Functions
}

\author{
Elena N. Meshcheryakova \\ Penza State Technological University, Penza, Russian Federation \\ *Corresponding author E-mail: murashkinaelena@mail.ru
}

\begin{abstract}
This article describes the possibility of triangulation function use for the classification, analysis and identification of complex microsystem physical object parameters. They analyzed the existing methods and identification algorithms, their advantages and disadvantages are highlighted. The existing methods of triangulation are considered, the possibility of Delaunay triangulation is described for surfactant signal 3-D model development and analysis. They developed the algorithm to identify the state of an object using the triangulation function that takes into account the change of node coordinates and the length of the triangulation grid edges. They presented the visual UML model. The conclusions are drawn about the possibility of triangulation function use for the analysis of complex microsystem state.
\end{abstract}

Keywords: micro and nanotechnologies, modeling, analysis, identification, algorithm, UML model, triangulation, surface-acoustic waves, complex microsystem, heterogeneous microstructures.

\section{Introduction}

One of the priority trends of RF science and technology development $[1,2]$ is the implementation of micro and nanotechnologies, which will allow to proceed to the implementation of complex microsystems. The physical basis for the development of complex microsystems is the microstructure combining the elements that have specific mechanical, electrical and optical properties. The development of structures with the combination of the listed properties is of particular complexity. Such structures can be called heterogeneous microstructures, uniting a set of physical objects with different physical properties in their composition.

The management of complex microsystems involves the determination of their parameters, which in its turn means the determination of physical object parameter values that form heterogeneous microstructures. The obtained values of physical object parameters should be structured in accordance with the purpose and the task of complex microsystem operation, as well as their structure and the functional purpose of elements. In order to determine the limitations of complex microsystems, it is necessary to monitor the parameters of physical objects.

Currently, surface acoustic waves (SAW) are used most widely for analysis, modeling, and data collection on the parameters of heterogeneous microstructures. SAW is an elastic wave that propagates in the upper surface layer along a half-space border or along the border of various physical nature media [17]. According to the type of distribution, there are SAWs with horizontal and vertical polarization. The type of SAW wave is determined primarily by the direction of the surface layer displacement along the $\mathrm{x}$ axis or along the $\mathrm{y}$ axis. The displacement of the surface layer is described not only by the direction, but also by the displacement of the matter particle oscillations in the sagittal plane $(\mathrm{x}, \mathrm{z})$, as a rule, the displacement has an elliptical shape.

In order to obtain a complete picture of the surface layer change, they developed the 3-D model of a physical object using the signals from the sensors on surface-acoustic waves. There is the task of identification, analysis and classification of the obtained 3 D model [20]. However, currently existing methods and algorithms describe the processes of 2-D signal classification, analysis and identification, the linear dimensions of which do not belong to the classification of "micro" and "nano". Therefore, in order to monitor the parameters of physical objects for complex microsystems, it is necessary to search for new methods of classification, analysis and identification of signals describing the state of objects.

In this paper, we simulated the identification algorithm for the 3-D model of a complex microsystem physical object using triangulation functions. The practical significance of the work is in the possibility of the obtained algorithm use to identify the changes in the physical parameters of complex microsystem elements, as well as to reduce the likelihood of recognition errors.

\section{Analysis of Existing Methods for Complex System Condition Monitoring}

The physical basis of complex microsystem existence is the heterogeneous microstructure, which is the combination of physical objects of various types. During the operation of complex microsystems, the parameters of physical objects are converted first into electrical parameters, and then into a digital code. Further, in accordance with the heterogeneous microstructure model, the resulting code table is structured according to the physical model, as well as with the consideration of existing limitations and physical meaning. The model of a complex microsystem is built on 
the basis of the physical model of a heterogeneous microstructure and in the general case includes some limitations on the setting of parameters and their derivatives, a target function or a tree of goals, as well as the functional or stochastic models determining the relationships between technical parameters.

In $[18,19]$, the complex microsystem is presented as the integration of microelectromechanical system with optical components. Proceeding from the results of technology current state analysis, it is necessary to expand the existing concepts of a heterogeneous microstructure and a complex microsystem.

Heterogeneous microstructure is a set of optical, electronic and mechanical properties of a physical object, combined into a single whole (Figure 1).

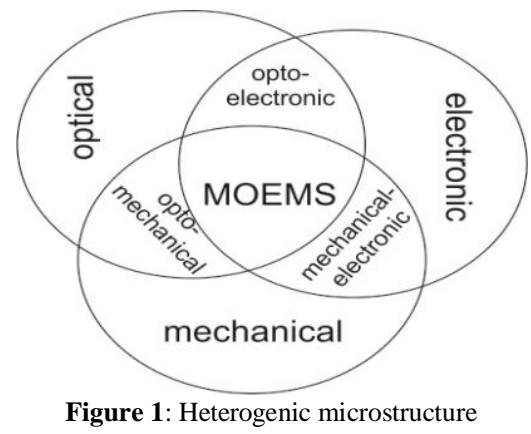

A complex microsystem is a set of information-controlling and functional elements united in one design model. The principle of structural element interaction is based on the physical model of a heterogeneous microstructure, taking into account the transitions to micro- and nano-elements. A complex microsystem takes into account the functional dependence of a physical object physical properties, but at the same time imposes certain restrictions on the actuating elements.

In order to determine the limitations of a complex microsystem, it is necessary to monitor the parameters of a physical object: input signal amplitude, frequency, phase, speed, etc. The combination of a physical object properties for a complex microsystem is determined by the analysis of the structural element physical effects. Nowadays, the following physical effects are most widespread during the development of a complex microsystem:

- inertia;

- gyroscopic effect;

- friction;

- deformation;

- electrical conductivity;

- thermal conductivity;

- phase transitions of the first and the second type;

- surface tension;

- sorption and diffusion.

However, the task of more and more functional complex microsystem development makes it necessary to conduct the research on such effects as the dispersion field, surface layer heterogeneity, and the thickness of structural elements [19]. The wavelength of the input signal and the thickness of the surface layer are of particular importance during the analysis of complex microsystems. The restrictions imposed on these parameters affect the nature of the input and the output signal oscillations, and, consequently, the geometric parameters of complex microsystem surface layer [24]. The study of such changes makes it possible to identify the occurrence of emergency situations in complex microsystems.

In the works [3-4] they consider the methods of identification system development for 2-D objects, which are the structural elements of complex systems. A particular attention is paid to the recognition process performance improvement and the probability increase. The identification algorithms for 2-D objects are described in detail by [5-7]. The most common method is the method of an object identification in "frequency-time" aspect.
Artificial neural networks are used as an identification tool. The features of neural network use in the problems of analysis and identification are considered in [22, 23, 25]. Figure 2 shows the generalized algorithm for the identification of complex system 2-D objects.

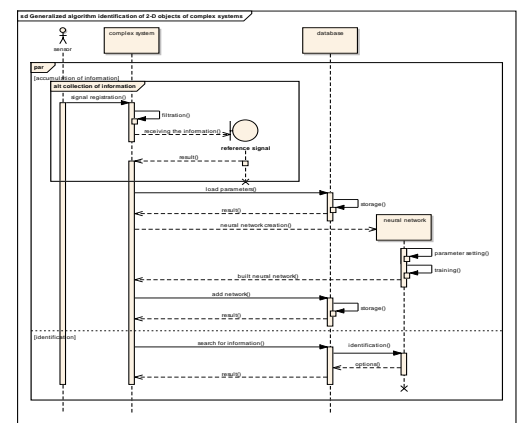

Figure 2: The generalized algorithm for complex system 2-D object identification

In order to obtain reliable information about the processes occurring in the structural elements of complex systems, the information about the reference signal is necessary. The sensor on the surface-acoustic waves reads the information from the elements. The system processes the received information from interference that may occur during a signal receiving and a signal transmission via communication channels. Taking into account the changes in the structure of the elements a neural network is developed. The neural network is trained on reference signals. Along with the process of the reference signal obtaining, the identification of the signal from the SAW sensor is carried out. The identification in this case is the process of the original and the reference signal comparison. In the event of a match, the conclusion is made about an emergency possibility.

To ensure a high probability of identification, it is necessary to accumulate information in the database. The accumulation involves the storage of information according to classification criteria that determine the likelihood of physical effects in the structural element of an object under study. In the works [8-10], they considered the methods of signal classification. Analyzing the works, we can conclude that in order to obtain reliable information it is necessary to use complete data sets (i.e., the data obtained over a long time).

Summarizing the results of the study, we can conclude that it is necessary to use artificial neural networks to identify the 3-D objects of complex microsystems. At the same time, the identification of an object in the frequency-time aspect is insufficient to display the full picture of object physical parameter change. Let us consider the possibility of triangulation function use to identify the 3-D objects of complex microsystems.

\section{Implementation of Triangulation Function to Identify the Condition of Complex Microsystems}

The algorithmic studies of triangulation development and application task appeared in the second half of the 20th century. From the mid-70-ies, they started the systematic study of geometric algorithms and the possibility of triangulation use in practical activity. Triangulation is the process of a plane division into $\mathrm{N}$ triangles. The use of triangulation is an integral part during the three-dimensional modeling of objects.

At the beginning of the XXI century, it was proposed to use the algorithmic dependence of the nodes and the edges of the triangulation grid to describe the changes in 3-D object parameters [26]. In order to perform the identification procedure, it was necessary to obtain a three-dimensional model of the object of study. In order to build three-dimensional models, two classes of 
triangulation methods are distinguished: direct and iterative. Iterative methods have sufficient versatility and can be used to triangulate the regions of arbitrary type [21], while they require greater labor intensity and implementation complexity for a particular structural element of a complex microsystem. It should be noted that the grids developed by iterative methods are unstructured and non-uniform. Unstructured nature is conditioned by the topology of the grid, which is formed in the process of development and can vary within one subarea. The quality of the grid is determined by its structure [21].

The algorithms of iterative triangulation method use in identification problems describe the dependence of the minimum triangulation density on the probability and identification speed $[11,14]$. The use of Delaunay triangulation greatly simplifies the task of the minimum density optimization. During the attempts to use the Delaunay triangulation for three-dimensional objects a number of problems is revealed: the grid does not maximize the minimum angles and sequential improvement algorithms do not work in space. The Canadian mathematician B. Joe proved that if new tetrahedra are added to an existing Delaunay triangulation (breaking one of the internal ones or attaching the tetrahedron to the outer face), then the resulting grid can be brought to the Delaunay triangulation using successive "trades" [26].

In order to develop the Delaunay triangulation, the source data is a set of points that should become the nodes of triangulation. An obvious advantage of this approach is an extremely precise control over the size of the grid elements. The dimensions of the grid elements are determined by the density of nodes. By increasing the density of node placement in particular places of the region, it is possible to achieve the local thickening of the grid near these features automatically. If the area is complex, you can ensure that nodes are placed on the surfaces and the edges of the constraints. The simplest and most frequently used node allocation method is based on the principles of boundary correction. The source area is placed in a super-region filled with the nodes in accordance with a given density of node placement, then the nodes lying near the border of the region are projected onto it, and the nodes outside the region are removed. There are other, more complex methods [12, $13,15,16]$. A common disadvantage of all methods based on the Delaunay criterion is a high sensitivity to the accuracy of machine calculations. The computational procedures used in these methods lead to the accumulation of rounding errors, which in the end can lead to the errors in the grid structure or an algorithm looping. The increase of calculation accuracy does not give significant results [21]. The use of numerical types with double precision stops working with the problems of medium complexity (the grids with several thousand nodes). A partial solution to this problem may be the use of fixed-point numeric types. It is also possible to use "accurate arithmetic", the implementation modules of which they have developed for many applied programming languages $(\mathrm{C}++$, Java, etc.).

Considering the peculiarities of the Delaunay triangulation implementation, we will conduct the simulation of the algorithm to identify the physical parameters of complex microsystem physical objects using UML 2.0. Figure 3 shows the developed algorithm.

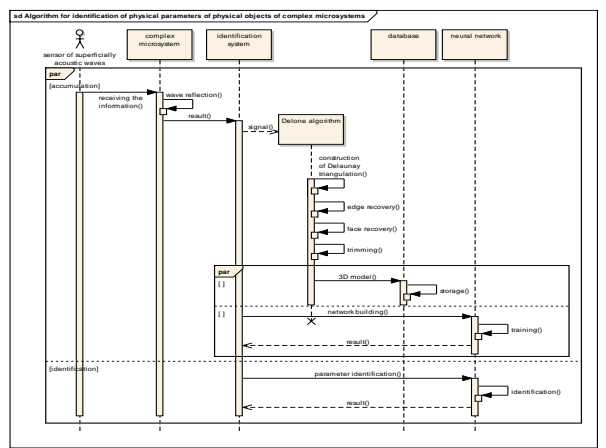

Figure 3: The algorithm for the identification of physical object parameter of complex microsystems
In order to obtain a high probability of physical parameter change recognition and, thus, the possibility of emergency situation occurrence, it is necessary to create the database of reference signals. For this, it is necessary to monitor the physical state of complex microsystem structural elements for a long time. The signal from the sensor to the SAW conducts the information retrieval from the microsystem, the signal is transmitted via the communication channel to the identification system. Using the Delaunay algorithm and the obtained SAW signals, they develop three-dimensional model describing the changes in the physical parameters of complex microsystem structural elements.

The essence of the algorithm consists in the Delaunay triangulation development without restrictions, and then the restoration of the surface and the restriction line takes place by the local restructuring of the grid. Delaunay algorithm can be divided into four stages:

1) the development of Delaunay triangulation without restrictions;

2) the restoration of restriction "edges";

3 ) the restoration of limitation surfaces;

4) the cutting off the "extra" tetrahedra that are outside the boundary of a given region.

Listing 1 shows the code of circle development.

Listing 1 - The building of circles

double strength $=$ sourceSl.getSignalStrength () ;

double distance $=$ calculateDistance(strength

*sourceSl.getDetector().getKoef());

MapCoordDbo coord = sourceAlert. getCoords();

Area area $=$ new Area (new Ellipse2F.Double $(\operatorname{coord} . g e t X()$, coord.get $\mathrm{Y}($ ), distance*2, distance*2));

The function of distance calculation between nodes is shown in Listing 2.

Listing 2 - The calculation of the distance between the nodes public static double calculation Distance (double db) \{

double [] $\mathrm{dbs}=\{\ldots\}$;

double [] $\mathrm{fts}=\{\ldots\}$;

double $\mathrm{k}=-\ldots$;

double prev $\mathrm{Db}=70$;

double prev Distant $=0$;

for (int $\mathrm{i}=0 ; \mathrm{i}<\mathrm{dbs}$. length; $i++)\{$

if $(\mathrm{dbs}[\mathrm{i}]<\mathrm{db})$ \{break;

koef $=($ dbs $[\mathrm{i}]-$ prev Db $) /($ fts $[\mathrm{i}]-$ prev Distant $)$;

prev $\mathrm{Db}=\mathrm{dbs}[\mathrm{i}]$;

prev Distant $=$ fts $[\mathrm{i}] ;\}$

return prev Distant + koef $*(\mathrm{db}-$ prev $\mathrm{Db}) ;\}$

Thus, we obtain the information on the coordinates of the triangulation grid nodes and on the coordinates and the length of the edges. This information is transmitted to the database for storage as standards. Then an artificial neural network is developed, which is trained on the standards. The information about network settings is stored in a database.

A distinctive feature of triangulation function use is the verification of the algorithm the computational stability [27]. The stability check includes:

1) The check of coincidence for two given points.

2) The check of the relative position of two points with respect to a straight line passing through two given points.

$\left(x_{1}-x\right)\left(y_{1}-y\right)-\left(x_{2}-x\right)\left(y_{2}-y\right)=0$

3 ) The check of collinearity for three given points.

4) The check of the relative position for a point and a triangle.

5) The check of bypass for three given points.

6) The check of the Delaunay condition fulfillment for two given adjacent triangles.

7) The localization of a point in triangulation.

8) The search for an intersection point of two straight lines.

In order to ensure the high stability of the computing ability of the algorithm, it is necessary to use the calculation accuracy of at least $10^{-5}$.

In parallel with the process of information accumulation, the sensor on SAW conducts the registration of physical state change concerning the structural elements of complex microsystems. 
Unlike the identification of 2D objects, the "non-conformity" search is conducted, i.e. the search for changes in the physical parameters of the object under study. If the obtained model does not coincide with the standard stored in the database, a signal is passed to the operator to make a decision. If these changes are not abnormal, the operator makes decisions about the making of these changes in the database as a possible deviation from the standard.

\section{Results}

The use of surface-acoustic waves makes it possible to analyze the physical state of the structural elements for complex microsystems, and increases the probability of change identification in the structure of the surface layer of the object under study. Using the signals from the sensors on surfactants and Delaunay triangulation, it was proposed to develop the 3-D model describing the changes in the physical parameters of structural elements. The developed identification algorithm recognizes the change in node coordinates and the length of the edges in the triangulation grid of the object under study. The use of triangulation increases the probability of recognition and makes it possible to use an algorithm to analyze the physical state of microsystems.

\section{Conclusions}

The developed algorithm is of practical interest in the issues of simulation modeling of physical object parameter identification in complex microsystems. Using the obtained results, it is planned to model and test an identification subsystem.

\section{Acknowledgments}

The work was prepared as the part of the grant for the scholarship from RF President to young scientists and graduate students engaged in advanced research and development within the priority areas of Russian economy modernization in 2018-2020, the project SP-5207.2018.5.

\section{References}

[1] (2016) Strategies for Scientific and Technological Development of the Russian Federation Presidential Decree from December 1, 2016 no. $642 \mathrm{ru} / \mathrm{acts} / \mathrm{bank} / 41449 \mathrm{http} / / / \mathrm{www} . \mathrm{kremlin}$.

[2] N.G. Kurakova, V.G. Zinov, L.A. Tsvetkova, "The system of measurable indicators for scientific and technological development of the Russian Federation: methodology and design" Scientific and Technical Information Processing, Volume 44, Issue 3, 1 July 2017, Pages 184-195.

[3] P.S. Prasad, Devi, B. Sunitha, Reddy M. Janga, V.K. Gunjan, "A survey of fingerprint recognition systems and their applications" Lecture Notes in Electrical Engineering, Volume 500, 2019, Pages 513-520, International Conference on Communications and Cyber Physical Engineering, ICCCE 2018; Hyderabad; India; 24 January 2018 through 25 January 2018; Code 217899

[4] K. Indira, K.V. Mohan, T. Nikhilashwary, "Automatic license plate recognition" Advances in Intelligent Systems and Computing, Volume 727, 2019, Pages 67-77, 1st International Symposium on Signal and Image Processing, ISSIP 2017; Kolkata; India; 1 November 2017 through 2 November 2017; Code 213529

[5] T. Walczak, J.K. Grabski, M.Michałowska, D. Szadkowska, "Application of artificial neural networks in the human identification based on thermal image of hands" Advances in Intelligent Systems and Computing, Volume 831, 2019, Pages 114122, International Conference of the Polish Society of Biomechanics, BIOMECHANICS 2018; Zielona Gora; Poland; 5 September 2018 through 7 September 2018; Code 216589

[6] S. Mane, G.Shah, "Facial recognition, expression recognition, and gender identification" Advances in Intelligent Systems and Computing, Volume 808, 2019, Pages 275-290, 2nd International
Conference on Data Management, Analytics and Innovation, ICDMAI 2018; Pune; India; 19 January 2018 through 21 January 2018; Code 217079

[7] P.S. Prasad, D. Baswaraj, "Iris recognition systems: A review" Lecture Notes in Electrical Engineering, Volume 500, 2019, Pages 521-527, International Conference on Communications and Cyber Physical Engineering, ICCCE 2018; Hyderabad; India; 24 January 2018 through 25 January 2018; Code 217899

[8] T.R. Vijaya Lakshmi, C. Venkata Krishna Reddy, "Object classification using sift algorithm and transformation techniques" Advances in Intelligent Systems and Computing, Volume 768, 2019, Pages 403-408, International Conference on Cognitive Informatics and Soft Computing, CISC 2017; Hyderabad; India; 20 December 2017 through 21 December 2017; Code 217069

[9] S. Schmidt, P.S. Heyns, "An open set recognition methodology utilising discrepancy analysis for gear diagnostics under varying operating conditions" Mechanical Systems and Signal Processing, Volume 119, 15 March 2019, Pages 1-22

[10] C.E. Ribeiro, L.E. Zárate, "Classifying longevity profiles through longitudinal data mining" Expert Systems with Applications, Volume 117, 1 March 2019, Pages 75-89

[11] Alihodzic, H. Smajlovic, E. Tuba, R. Capor Hrosik, M. Tuba, "Adjusted artificial bee colony algorithm for the minimum weight triangulation" Advances in Intelligent Systems and Computing, Volume 741, 2019, Pages 305-317, 4th International Conference on Harmony Search, Soft Computing and Applications, ICHSA 2018; Gurgaon; India; 7 February 2018 through 9 February 2018; Code 217459

[12] Shan, N. Ren, Z. Xue, "Stereovision-based surface deformation detection of offshore wind turbine model under ship impact" Measurement: Journal of the International Measurement Confederation, Volume 131, January 2019, Pages 605-614

[13] J.B. Rodrigues, A.V.M. Ferreira, I.M.O. Maia, G. Braz Junior, J.D.S. de Almeida, A.C. de Paiva, "Image Processing of Artworks for Construction of $3 \mathrm{D}$ Models Accessible to the Visually Impaired" Advances in Intelligent Systems and Computing, Volume 793, 2019, Pages 243-253, Joint proceedings of the AHFE International Conference on Advanced Production Management and Process Control, AHFE International Conference on Human Aspects of Advanced Manufacturing, and AHFE International Conference on Additive Manufacturing, Modeling Systems and 3D Prototyping, 2018; Orlando; United States; 21 July 2018 through 25 July 2018; Code 215429

[14] P.S. Prasad, D. Baswaraj, "Iris recognition systems: A review" Lecture Notes in Electrical Engineering, Volume 500, 2019, Pages 521-527, International Conference on Communications and Cyber Physical Engineering, ICCCE 2018; Hyderabad; India; 24 January 2018 through 25 January 2018; Code 217899

[15] J. Liu, D. Bai, L. Chen, "3-D point cloud registration algorithm based on greedy projection triangulation" Applied Sciences (Switzerland), Volume 8, Issue 10, 30 September 2018, Article number 1776

[16] Alonazi, Y. Ma, R. Tafazolli, "Delaunay triangulation based interpolation for radio map construction with reduced calibration" 2017 9th IEEE-GCC Conference and Exhibition, GCCCE 2017, 27 August 2018, Article number 8448199, 9th IEEE-GCC Conference and Exhibition, GCCCE 2017; Bahrain Gulf Conference and Exhibition CenterManama; Bahrain; 8 May 2017 through 11 May 2017; Category numberCFP1729A-ART; Code 139101

[17] Surface acoustic waves in radio engineering / comp. by G.F. Afanasyev, L.Sh. Biktimirov. - Ulyanovsk: UISTU, 2013. - 75 p.

[18] Konoplev B.G., Lysenko I.E. The components of microsystem technology. Part 1.- Taganrog: TTI SFU Publishing House, 2009.$117 \mathrm{p}$.

[19] Yankin S.S. The interaction of surface acoustic waves with inhomogeneities comparable to the wavelength: diss. by the Cand. of Phys.-math. sciences. - Saratov, 2015. - 117 p.

[20] Mikheev M.Yu., Remontov A.P., Mescheryakova E.N., Kosolapov V.V., Astakhova T.N. The use of Wolfram Mathematica technology for surface-acoustic wave modeling. NGIEI Bulletin. 2017. - No. 9 (76). - pp. 16-23.

[21] Galanin M.P., Scheglov I.A. The development and the implementation of three-dimensional triangulation algorithms for complex spatial domains: iterative methods M., 2006. - 35 p.

[22] M.Yu. Mikheev, T.V. Zhashkova, E.N. Meshcheryakova, K.V. Gudkov, A.K. Grishkoб "Imitation modelling for the subsystem of identification and structuring data of signal sensors" Proceedings of 
2016 IEEE East-West Design and Test Symposium, EWDTS 2016, 2016. p. 7807748

[23] Murashkina E.N., Mikheev M.Yu. The development of simulation models of information signal identification and structuring subsystem from the sensors on surface-acoustic waves // Proceedings of the international symposium "Reliability and quality". - 2015. - V. 1. - pp. 187-190.

[24] Murashkina E.N., Mikheev M.Yu. Simulation modeling of neural network identification for complex shape signals. // Proceedings of the International Symposium "Reliability and Quality". - 2014. - V. 1. - pp. 203-206.

[25] Murashkina E.N., Zhashkova T.V. Identification of critical conditions for the system of monitoring and control // Modern high technologies. - 2014. - № 5-2. - pp. 62.

[26] B. Joe. Construction of Three-Dimensional Delaunay Triangulations Using Local Transformations // Computer Aided Geometric Design, Vol. 8, pp. 123-142, 1991

[27] Skvortsov A.V. Delaunay triangulation and its application. Tomsk: Tom. University Publishing house, 2002. - 128 p. 